\title{
Estimating the impact of COVID-19-induced coagulopathy
}

Ashwin Subramaniam, ${ }^{1,2,3}$ FCICM, Muhammad Alamgeer, ${ }^{4,5}$ FRACP

The current coronavirus disease 2019 (COVID-19) pandemic has exerted significant strain on healthcare worldwide. Mostly asymptomatic or mildly symptomatic, COVID-19 caused by SARS-CoV-2 is described as a thrombo-inflammatory syndrome, ${ }^{1}$ with severe respiratory illness occurring in about $13 \%$ of affected patients. This can rapidly transform into a lifethreatening condition in about $4 \%$ of cases (particularly those with comorbidities), characterised by severe acute respiratory distress syndrome, septic shock, metabolic acidosis and coagulopathy/disseminated intravascular coagulopathy (DIC). ${ }^{1}$ A proportion of patients develop severe coagulopathy, termed COVID19-induced coagulopathy (CIC), which is one of the leading causes of mortality in these patients. The exact mechanism of CIC is still poorly understood; it is postulated as a complex thrombo-inflammatory process, resulting from the host viraemic response, leading to dysregulated coagulation due to diffuse endothelial dysfunction. It ultimately triggers the formation of complement-induced thrombosis, resulting in systemic microangiopathy and thromboembolism, and DIC. ${ }^{2}$ Due to the high mortality, early identification of clinical and laboratory predictors of disease severity is imperative to develop and gauge adequate interventions to reduce health and economic burden.

In this issue of the Annals, Mitra and colleagues ${ }^{3}$ present a rigorous and comprehensive systematic review and meta-analysis of the natural history of CIC. The review included 26 studies involving 5,243 adult patients with severe COVID-19, published from 6 different countries. ${ }^{3}$ The authors aimed to identify coagulation parameters (platelets, D-dimer, prothrombin time, activated partial thromboplastin time and fibrinogen) that could predict and prognosticate the severity of the disease progression.

The included studies were observational and lacked comprehensive global representation, with the majority (20 of the 26) studies coming out of China alone and the rest (6/26) from Europe and North America. Nonetheless, they were rated to be of the highest quality (score $>6 / 9$ using Joanna Briggs Institute checklist). The authors used appropriate methods to pool estimates in the presence of high heterogeneity and their results were robust to a variety of sensitivity analyses and under a diverse set of assumptions.

Mitra et al. observed that $30.1 \%$ of patients had severe coagulopathy (95\% confidence interval [CI] 21.8-39.1), with a pooled mortality estimated from 15 studies of $14.0 \%$ (95\% CI 8.4-20.7). They also reported significant differences in the pooled mean in the blood levels of coagulation parameters (platelets, D-dimer, prothrombin time and fibrinogen) among patients with non-severe and severe disease. They also identified differences according to other demographics. Older patients, male sex, and those with comorbidities (e.g. diabetes mellitus, hypertension or cardiovascular disease) had a higher likelihood of progression to severe disease with coagulopathy. Consistent with the evolving data, lower platelet counts, and higher D-dimer levels correlated with disease severity.

These findings have important clinical and epidemiological implications. Primarily, the results were representative of the patient demographics, clinical presentation, disease severity and mortality rates early in the pandemic. ${ }^{4}$ Moreover, the information may help the ongoing pandemic planning, resource allocation, and estimation of both the health-related and socioeconomic impact of COVID-19. ${ }^{5}$ Finally, the poor clinical outcome among older patients highlight the importance of preventing further outbreaks among the extremely vulnerable group. The results illustrate useful methods for estimating risk across multiple studies during an evolving pandemic. This would help the design of future studies of hospitalised patients with COVID-19 by providing a more precise estimate of severe coagulopathy risk.

\footnotetext{
${ }^{1}$ Department of Intensive Care Medicine, Peninsula Health, Frankston, Victoria, Australia

${ }^{2}$ Department of Epidemiology and Preventive Medicine, School of Public Health and Preventive Medicine, Monash University, Melbourne, Victoria, Australia

${ }^{3}$ Department of Intensive Care Medicine, The Bays Hospital, Mornington, Victoria, Australia

${ }^{4}$ Department of Medical Oncology, Monash Health, Clayton, Victoria, Australia

${ }^{5}$ Department of Haematology and Oncology, The Bays Hospital, Mornington, Victoria, Australia

Correspondence: Dr Ashwin Subramaniam, Department of Intensive Care, Frankston Hospital, 2 Hastings Road, Frankston, Victoria 3199 , Australia.

Email: ashwin.subramaniam@monash.edu / Muhammad.alamgeer@monash.edu
} 
There were some inherent limitations.

Firstly, Mitra et al. only report analysis based mainly on retrospective or cohort studies during the early pandemic. ${ }^{3}$ However, recent reviews on thromboinflammatory and haematological biomarkers have also revealed analogous findings that patients with severe COVID-19 manifest hypercoagulable conditions (e.g. elevated D-dimer and fibrinogen) as well as a drop in platelet counts. ${ }^{2,6}$ It is expected that results from new studies are unlikely to alter the key findings of Mitra et al. and therefore, their results are possibly generalisable.

Secondly, the authors report significant heterogeneity. There are many causes of heterogeneity across studies: variations in case-mix across cohorts, hospital admission criteria across regions, a maximum time of outcome recording, and whether a longer follow-up was available. However, the Grading of Recommendation, Assessment, Development, and Evaluation classification that the authors used demonstrated a moderate to high level of certainty, hence enhancing the reliability of the results. Furthermore, even across the leave-one-out sensitivity analysis, there were no outliers in patients with severe COVID-19.

Thirdly, the authors could not determine the timing of these laboratory tests in relation to the clinical disease course. This information would be vital to understand the impact of trends in changing coagulation profiles during the disease process and outcomes.

Fourthly, most of the reported studies pre-date the RECOVERY study, or Randomised Evaluation of COVID-19 Therapy. ${ }^{7}$ The use of corticosteroids has been linked with an increased risk of venous thromboembolic disease. ${ }^{8}$ However, low-dose corticosteroid use in pro-inflammatory conditions has caused a reduction in fibrinogen and procoagulant factors, and an increase in anticoagulant factors. ${ }^{9}$ The use of corticosteroids was not reported by Mitra et al. in their review. The potential antithrombotic properties in patients with deranged coagulation profile and low-dose dexamethasone warrant further investigation.

Fifthly, there are differences in reporting of disease severity across regions. This could be explained by causal mechanisms but could alternatively arise due to variations in case definitions, confounded by differences in disease susceptibility across age group, sex and presence of comorbidities; healthcare structure; and a possible selection bias created by decisions to provide or withhold aggressive treatments. Standardised reporting and adjustment for such potential confounders would most likely provide reliable comparisons not only across populations, but also with individual patients.

Finally, a recent observational study has linked systemic anticoagulation with significantly improved survival in mechanically ventilated patients with COVID-19. ${ }^{10}$ However, there is no concrete evidence on when to use therapeutic anticoagulation.

Future studies should focus on stratifying all patients with COVID-19 who had coagulation profiles upon admission to the intensive care unit (ICU), followed by another on ICU discharge or death. This could show a correlation with highly abnormal coagulation profile before death, independent of the treatment received, and demonstrate that deranged coagulation leads to impending death. Furthermore, it will be essential to incorporate a CIC-specific scoring system, rather than extrapolating results using the DIC score, ${ }^{11}$ to guide therapies in patients with CIC.

Overall, the systematic review and meta-analysis from Mitra et al. reinforce that patients with severe COVID-19 are likely to have a deranged coagulation profile. This profile is associated with poorer outcomes, and dramatically increases among older age groups. One could postulate that over time, COVID-19 vaccination will protect people from getting sick or severely ill with the disease, and may reduce CIC. Until then, these results highlight an urgent need to identify new prognostication tools and treatment options to reduce mortality and prevent the disease from worsening and spreading.

\section{REFERENCES}

1. Ciceri F, Beretta L, Scandroglio AM, et al. Microvascular COVID-19 lung vessels obstructive thromboinflammatory syndrome (MicroCLOTS): an atypical acute respiratory distress syndrome working hypothesis. Crit Care Resusc 2020;22:95-7.

2. Chaudhary R, Garg J, Houghton DE, et al. Thrombo-inflammatory Biomarkers in COVID-19: Systematic Review and Meta-analysis of 17,052 patients. Mayo Clin Proc Innov Qual Outcomes 2021. https://doi.org/10.1016/j.mayocpiqo.2021.01.009

3. Mitra S, Ling RR, Yang IX, et al. Severe COVID-19 and coagulopathy: A systematic review and meta-analysis. Ann Acad Med Singap 2021;50:325-35.

4. Zhou F, Yu T, Du R, et al. Clinical course and risk factors for mortality of adult inpatients with COVID-19 in Wuhan, China: a retrospective cohort study. Lancet 2020;395:1054-62.

5. Abdollahi E, Champredon D, Langley JM, et al. Temporal estimates of case-fatality rate for COVID-19 outbreaks in Canada and the United States. CMAJ 2020;192:E666-70. 
6. Asghar M, Hussain N, Shoaib H, et al. Hematological characteristics of patients in coronavirus 19 infection: a systematic review and metaanalysis. J Community Hosp Intern Med Perspect 2020;10:508-13.

7. The RECOVERY Collaborative Group. Dexamethasone in Hospitalized Patients with Covid-19 - Preliminary Report. N Engl J Med 2021. doi: 10.1056/NEJMoa2021436

8. Johannesdottir SA, Horváth-Puhó E, Dekkers OM, et al. Use of glucocorticoids and risk of venous thromboembolism: a nationwide population-based case-control study. JAMA Intern Med 2013; 173:743-52.
9. van Zaane B, Nur E, Squizzato A, et al. Systematic review on the effect of glucocorticoid use on procoagulant, anti-coagulant and fibrinolytic factors. J Thromb Haemost 2010;8:2483-93.

10. Paranjpe I, Fuster V, Lala A, et al. Association of Treatment Dose Anticoagulation With In-Hospital Survival Among Hospitalized Patients With COVID-19. J Am Coll Cardiol 2020; 76:122-4.

11. Taylor FB Jr, Toh CH, Hoots WK, et al. Towards definition, clinical and laboratory criteria, and a scoring system for disseminated intravascular coagulation. Thromb Haemost 2001;86:1327-30. 